Cette étude a fait l'objet d'une thèse de Doctorat d'Université présentée à la Faculté des Sciences de Paris (1967) par le premier auteur. Elle doit être reprise sur un effectif plus important en tenant compte d'un effet maternel possible sur le format des animaux en raison des différences raciales de prolificité.

\title{
VARIATION DES BESOINS D'ENTRETIEN ET DE CROISSANCE CHEZ LES JEUNES LAPINS (42 A 84 JOURS) DE DEUX RACES
}

R. Rouvier. - Station de Génétique quantitative et appliquée, C.N.R.Z., 78-Jouy-en-Josas.

Cinquante-huit lapins mâles de race Argenté de Champagne et 40 de race Grand Russe ont été alimentés individuellement entre le sevrage à 42 jours d'âge et l'abattage à 84 jours. L'aliment granulé, dosait de 13,4 à $14,7 \%$ de matière azotée totale, et, de l'ordre de 14,5\% de cellulose. L'indice de consommation moyen est légèrement plus élevé pour les Argenté de Champagne $(4,46)$ que pour les Grands Russes $(4,19)$. Cependant, le poids moyen des Argenté de Champagne dans la période considérée est plus élevé, et la composition anatomique des animaux à l'abattage est différente : rapport muscle los égal à 4,5 chez les Argenlé de Champagne et 4,0 chez les Grands Russes, pourcentage du tissu gras disséqué de la carcasse égaux respectivement à 7,1 et $5,9 \%$. Les coefficients de l'équation de régression de la quantité d'aliment consommé par jour sur le gain de poids journalier moyen (coefficient $a_{1}$ ) et le poids moyen de l'animal (coefficient $a_{2}$ ) pendant la période considérée, ont été obtenus pour chacune des deux races. L'hypothèse de deux plans de régression parallèles est acceptable, et l'on a obtenu les estimations de leurs valeurs communes : $a_{1}=2,43 \pm 0,19$ et $a_{2}=0,025 \pm 0,012$ avec $R^{2}=0,60$.

Les trois variables poids total du muscle ou de gras, ou poids du gras périrénal, considérées successivement comme variables prédictrices supplémentaires, présentent approximativement le même intérêt. Ici aussi l'hypothèse de deux plans de régression parallèles est acceptable. Le carré de la corrélation multiple entre la quantité d'aliment consommé d'une part, le gain de poids journalier moyen, le poids moyen, le poids de gras périrénal d'autre part est de 0,70 . Ces résultats fournissent des premières estimations des besoins de croissance et d'entretien moyens. Les équations obtenues permettent de comparer les efficacités de l'utilisation alimentaire des animaux des deux races, pour un même gain de poids et poids moyen, ainsi que pour une même composition anatomique. Les résultats obtenus lors de ces comparaisons indiquent l'intérêt de la prise en compte de la composition anatomique. 